\title{
Conditions for successful extended inflation
}

\author{
Anne M. Green and Andrew R. Liddle \\ Astronomy Centre, University of Sussex, Falmer, Brighton BN1 9QH, U. K.
}

(August 20, 2018)

\begin{abstract}
We investigate, in a model-independent way, the conditions required to obtain a satisfactory model of extended inflation in which inflation is brought to an end by a first-order phase transition. The constraints are that the correct present strength of the gravitational coupling is obtained, that the present theory of gravity is satisfactorily close to general relativity, that the perturbation spectra from inflation are compatible with large scale structure observations and that the bubble spectrum produced at the phase transition doesn't conflict with the observed level of microwave background anisotropies. We demonstrate that these constraints can be summarized in terms of the behaviour in the conformally related Einstein frame, and can be compactly illustrated graphically. We confirm the failure of existing models including the original extended inflation model, and construct models, albeit rather contrived ones, which satisfy all existing constraints.
\end{abstract}

PACS numbers: 98.80.Cq Sussex preprint SUSSEX-AST 96/4-1, astro-ph/9604001

\section{INTRODUCTION}

The extended inflation scenario [1,2] offers the prospect of resuscitating the original idea of Guth [3] that inflation [4 [6] could be driven by a metastable vacuum energy and end by the tunnelling of the associated scalar field to the true minimum of its potential. The strategy is to implement inflation in an extended theory of gravity, such as a scalar-tensor theory, in which the expansion rate induced by a vacuum energy is slower than exponential. Under such circumstances, one is guaranteed that the phase transition will be able to successfully complete, which proves not to be the case in Einstein gravity if sufficient inflation is demanded to solve the usual cosmological problems.

Moving to an extended gravity theory is an interesting way of generalizing existing inflation models, because such scenarios are much more highly constrained [7] than alternative generalizations where extra scalar fields are added by hand. In particular, one knows that the theory must mimic general relativity to a high degree at the present epoch, and there are further strong constraints on the variation of the strength of the gravitational interaction going all the way back to the time of nucleosynthesis. These additional constraints naturally have the effect of making it more difficult to obtain a viable model.

When one chooses to end inflation via a first-order phase transition, where bubbles of true vacuum nucleate, expand and coalesce, this introduces further constraints, because it is possible for the earliest true-vacuum bubbles which nucleate to be caught up in the subsequent inflationary expansion and stretched to astrophysically large sizes 8.96. These can contribute both density perturbations and microwave background anisotropies over and above those caused by quantum fluctuations [10] as in all inflationary models 11]. Near the general relativity limit, the distribution of bubbles is scale-invariant (in the sense of equal volume residing in bubbles within a given logarithmic size interval) which is far from acceptable [8,9].

The original extended inflation model [1] was implemented in the Jordan-Brans-Dicke (JBD) theory of gravity [12,7], where the gravitational 'constant' is replaced by a field $\Phi$ whose variation is controlled by a coupling parameter $\omega$. General relativity is obtained in the limit of large $\omega$. In that model, it was quickly shown that the competing needs of staying close to the general relativity limit to match present observations $(\omega>500$ [13, , $]$ ), and of obtaining a satisfactory bubble distribution $(\omega \lesssim 25$ (8.9), are mutually exclusive. This became known as the big-bubble problem, and various strategies have been brought into play in an attempt to evade it. The simplest is to introduce a mechanism which invalidates the present-day bound on $\omega$; this can for example be achieved by introducing a potential for the Brans-Dicke field $\Phi$ which is negligible during inflation and which prevents its variation at the present epoch. Alternatively, one can move to a general scalar-tensor theory, in which $\omega$ is allowed to depend on $\Phi$, which allows one to exercise control over how closely the general relativity limit is attained at different epochs.

Both these strategies have more recently suffered further constraints, under the assumption that the quantum fluctuations during inflation provide the density perturbations which are responsible for large-scale structure and microwave background anisotropies. The results of COBE in combination with large-scale structure studies quickly led to the conclusion that the spectrum of density perturbations must not be too far from scale-invariant, if the observed structures are to be reproduced. However, in extended inflation models one expects that if one makes the necessary moves to break the scale-invariance of the bubble distribution, then one will also destroy the scale-invariance of the density perturbation spectrum. This implies two opposing constraints, but now both to be applied during inflation. This extra consideration 
proved sufficiently stringent to exclude all models in the existing literature in which inflation ends by nucleation [14,15,

In the literature, a substantial number of models falling into the extended inflation class have been devised 17, 16, 18, and examined on a more or less case by case basis [19:20]. In this paper, we shall place the constraints in a more general framework, allowing one to see easily the problems of existing models. As a by-product, this will enable us to construct working models satisfying all present constraints, though as we shall see the constraints combine in such a way as to make such models appear extremely contrived. The prognosis for the extended inflation scenario therefore continues to look poor.

\section{EXTENDED INFLATION MODELS}

We work within a class of theories featuring a general scalar-tensor theory with Brans-Dicke field $\Phi$, plus a separate scalar field $\sigma$ trapped in a metastable state with energy density $V_{0}$. Models of this sort were discussed in Refs. [18,20]. The action is

$$
\begin{gathered}
S=\int \mathrm{d}^{4} x \sqrt{-g}\left\{\frac{m_{\mathrm{Pl}}^{2}}{16 \pi}\left[\Phi R-\frac{\omega(\Phi)}{\Phi} \partial_{\mu} \Phi \partial^{\mu} \Phi+2 \Phi \lambda(\Phi)\right]\right. \\
\left.-\frac{1}{2} \partial_{\mu} \sigma \partial^{\mu} \sigma+V(\sigma)\right\} .
\end{gathered}
$$

We have normalized the Brans-Dicke field by pulling out a pre-factor; with these conventions $\Phi$ is dimensionless and its present value is 1 . The function $\lambda(\Phi)$ corresponds to a potential for the Brans-Dicke field, which we shall assume to be negligible during inflation. We need assume nothing about the potential for $\sigma$ beyond that the metastable state has potential $V_{0}$ and that the field can tunnel from this to the true vacuum.

A vital tool for studying this theory is the conformal transformation [21], which simplifies the gravitational sector to general relativity. On performing the conformal transformation $g_{\mu \nu}=\Omega^{-2} \tilde{g}_{\mu \nu}$ with $\Omega=\sqrt{\Phi}$, and defining a new scalar field by

$$
\phi \equiv \frac{m_{\mathrm{Pl}}}{\sqrt{8 \pi}} \int \frac{\mathrm{d} \Phi}{\Phi} \sqrt{\omega(\Phi)+\frac{3}{2}}
$$

one obtains the equivalent action

$$
\begin{array}{rl}
S=\int \mathrm{d}^{4} & x \sqrt{-\tilde{g}}\left[\frac{m_{\mathrm{Pl}}^{2}}{16 \pi} \tilde{R}-\frac{1}{2} \partial_{\mu} \phi \partial^{\mu} \phi\right. \\
& \left.+2 U(\phi)-\frac{1}{2 \Omega^{2}} \partial_{\mu} \sigma \partial^{\mu} \sigma+\frac{V(\sigma)}{\Omega^{4}}\right]
\end{array}
$$

\footnotetext{
${ }^{*}$ There are however models such as hyperextended inflation [16], in which inflation is brought to an end through dynamical evolution, with bubbles then nucleating in the postinflationary phase.
}

where $U(\phi) \equiv \lambda(\Phi) m_{\mathrm{Pl}}^{2} / 16 \pi \Phi$. Here 'tilde' indicates quantities in the transformed frame, which we refer to as the Einstein frame (the original one being the Jordan frame). The field $\phi$ has been defined so as to have a canonical kinetic term. Assuming $\lambda(\Phi)$ vanishes and that the $\sigma$ field is in its trapped phase, this simplifies to

$$
S=\int \mathrm{d}^{4} x \sqrt{-\tilde{g}}\left[\frac{m_{\mathrm{Pl}}^{2}}{16 \pi} \tilde{R}-\frac{1}{2} \partial_{\mu} \phi \partial^{\mu} \phi+V_{0} \Omega^{-4}\right]
$$

This is the action for a standard single field chaotic inflation model [4] with potential

$$
V(\phi)=V_{0} \Omega^{-4}=V_{0} \Phi^{-2},
$$

where $\Phi(\phi)$ is given by Eq. (2). A change in the coupling function $\omega(\Phi)$ in the Jordan frame therefore leads to a different potential $V(\phi)$ in the Einstein frame, by changing the relation between $\phi$ and $\Phi$. Equally, if we are given a potential $V(\phi)$ in the Einstein frame, there exists a corresponding Jordan frame theory with a trapped field $\sigma$ and a coupling function $\omega(\Phi)$. The only difference is that in the standard chaotic inflation scenario inflation ends when the potential becomes too steep to maintain inflation, while in our scenario inflation may also end when the $\sigma$ field tunnels, which can happen at any location on the potential $V(\phi)$.

Our strategy is to use the Einstein frame scalar field $\phi$ as a time variable, even when referring to quantities in the Jordan frame. The bulk of the analysis shall be carried out in the Einstein frame, using the usual slowroll approximation. In the Einstein frame, we define the slow-roll parameters 14

$$
\begin{aligned}
\epsilon(\phi) & =\frac{m_{\mathrm{Pl}}^{2}}{16 \pi}\left(\frac{V^{\prime}}{V}\right)^{2} ; \\
\eta(\phi) & =\frac{m_{\mathrm{Pl}}^{2}}{8 \pi} \frac{V^{\prime \prime}}{V},
\end{aligned}
$$

where primes indicate derivatives with respect to $\phi$. Inflation occurs in the Einstein frame provided these are much less than one. The number of $e$-foldings of inflation in the Einstein frame, between two values of $\phi$, is given by the usual formula

$$
N\left(\phi_{1}, \phi_{2}\right) \simeq-\frac{8 \pi}{m_{\mathrm{Pl}}^{2}} \int_{\phi_{1}}^{\phi_{2}} \frac{V}{V^{\prime}} \mathrm{d} \phi .
$$

From Eqs. (2) and (5), one can derive [22] a remarkably simple relation between $V(\phi)$ and $\omega(\Phi)$

$$
\omega(\Phi)+\frac{3}{2}=\frac{2}{\epsilon(\phi)} .
$$

In the particular case of the JBD theory where $\omega$ is constant, then $\epsilon$ is constant too and we have the well known result of power-law inflation in the Einstein frame. 


\section{OBSERVATIONAL CONSTRAINTS}

Here we go through the constraints on the scenario.

\section{A. Present strength of gravity}

To reproduce the present value of the gravitational coupling, we require $\Phi=1$ at the present. If $\Phi$ is a free field, then the present strength of gravity is determined dynamically, by whatever $\Phi$ happens to have evolved to. During conventional evolution such as matter domination or radiation domination, the variation of $\Phi$ is extremely slow 23.8], unless $\omega$ is very small. Consequently, it is an excellent approximation to assume that the present value is the same as that at the end of inflation. In that case $V_{0}$ can be identified with the Einstein frame potential energy at the end of inflation.

If instead there is a potential for $\Phi$, its value at the end of inflation becomes unimportant and instead we require that the minimum of the potential be at $\Phi=1$; the present gravitational coupling is therefore determined by the parameters of the theory. In that case, the value of $\Phi$ at the end of inflation can effectively be incorporated into the amplitude of $V(\phi)$, by writing

$$
V(\phi)=\frac{V_{0}}{\Phi_{\mathrm{end}}^{2}}\left(\frac{\Phi_{\mathrm{end}}}{\Phi}\right)^{2} .
$$

Then the potential energy at the end of inflation in the Einstein frame is $V_{0} / \Phi_{\text {end }}^{2}$.

In the following expressions, we shall assume a free $\Phi$ field so that $V\left(\phi_{\text {end }}\right)=V_{0}$. The introduction of a potential for $\Phi$ can be simply accounted for via the scaling above.

\section{B. Recovering general relativity}

If the $\Phi$ field is a free field, then present-day limits from solar system observations demand that $\omega>500$ [13,7], with a comparable constraint from nucleosynthesis [24]. (There is also a limit on $\mathrm{d} \omega / \mathrm{d} \Phi$, but typically this is much weaker.) This constraint is extremely difficult to satisfy within the extended inflation context - we see from Eq. (9) that it requires $\epsilon(\phi)$ at the end of inflation to be less than $1 / 250$. This tough constraint can be evaded by the introduction of a potential for the $\Phi$ field, which prevents its variation at the low energy scales of our present universe [9] and permits any present value of $\omega$.

\section{The bubble spectrum}

Bubble nucleation is typically discussed in the Jordan frame. The nucleation rate per unit volume per unit time
$\Gamma$ is a constant determined by the shape of the potential barrier between the false and true vacuum states (see e. g. Ref. [5]), but the quantity of interest is not this, but rather the nucleation rate per Hubble volume per Hubble time

$$
E=\frac{\Gamma}{H^{4}},
$$

where $H$ is the Jordan frame Hubble parameter. Once $E$ exceeds some critical value of order unity, the phase transition is able to complete [25]. We shall assume that the critical value is unity, in which case $\Gamma=H_{\mathrm{end}}^{4}$.

The bubbles which are potentially constraining are those which are stretched to large sizes by the subsequent inflationary expansion, so they would be nucleated some time before the end of inflation. For typical parameters, a bubble of present size $20 h^{-1} \mathrm{Mpc}$ would have nucleated about $55 e$-foldings from the end . The crucial quantity is therefore the nucleation rate at that time, which we shall denote by $E_{55}$. Original calculations of the bubble spectrum 8, 9, 26] were combined with fairly ad hoc observational criteria to obtain the constraint $\omega \lesssim 25$ on the original extended inflation model. These calculations were followed up by more specific ones 27. which included detailed calculations of the effect of bubbles on the microwave background; it was estimated that any bubble larger than $20 h^{-1} \mathrm{Mpc}$ would be seen in the microwave background, and integration over the bubble spectrum leads to a slightly stronger version of the constraint.

However, it is possible to adopt a much more straightforward approach which captures the essence of the constraint, which is simply to limit the value of $E$ at the time the dangerous bubbles are forming. Because $E$ is growing with time, the most constraining bubbles are always the smallest ones that you can see, and so it is reasonable to take the constraint as being on the instantaneous value of $E$ when those bubbles formed, i.e. $E_{55}$. This approach was adopted in Ref. [28], where it was shown present observations demand

$$
E_{55}=\left(\frac{H_{\mathrm{end}}}{H_{55}}\right)^{4}<10^{-5} .
$$

This constraint is extremely conservative, since many very generous assumptions were made in computing the effect of bubbles on the microwave background [27].

We need to compute the Jordan frame Hubble parameter in terms of the Einstein frame quantities. Continuing to use 'tilde' to indicate Einstein frame quantities, the transformation rule

$$
\mathrm{d} t=\Omega^{-1} \mathrm{~d} \tilde{t} \quad ; \quad a(t)=\Omega^{-1} \tilde{a}(\tilde{t})
$$

\footnotetext{
${ }^{\dagger}$ It doesn't really matter in which frame the $e$-foldings are computed; see the Appendix.
} 
implies, continuing to measure time with the Einstein frame scalar field, that

$$
H(\phi)=\Omega \tilde{H}-\frac{\mathrm{d} \Omega}{\mathrm{d} t} .
$$

Some algebra brings us to the result

$$
H(\phi)=\left(\frac{8 \pi}{3 m_{\mathrm{Pl}}^{2}}\right)^{1 / 2} V_{0}^{1 / 4} V^{1 / 4}(\phi)\left[1-\frac{\epsilon(\phi)}{2}\right] .
$$

Notice that the Jordan frame Hubble parameter only goes as the fourth root of the Einstein frame potential, whereas the Einstein frame Hubble parameter goes as the square root. From this expression we obtain

$$
\frac{H(\phi)}{H_{\mathrm{end}}}=\left(\frac{V(\phi)}{V_{0}}\right)^{1 / 4}\left[\frac{1-\epsilon / 2}{1-\epsilon_{\mathrm{end}} / 2}\right] .
$$

Unless the $\epsilon$ terms are anomalously large, the square bracketed term can be taken as unity, and the bubble constraint translated into the Einstein frame becomes simply

$$
\frac{V\left(\phi_{55}\right)}{V_{0}} \geq 10^{5}
$$

\section{Density perturbations}

Because we know that general relativity is a good description of the present universe, we know that the Jordan and Einstein frames must coincide to high accuracy at the present. Therefore calculations can be carried out in whichever frame is easiest. We use the Einstein frame.

Inflation models produce spectra of both density perturbations and gravitational waves. It is natural to assume that these perturbations are those responsible for structure in the universe, and under that assumption their form is strongly constrained by a variety of observations. The general scalar-tensor case, where both the inflaton and Brans-Dicke fields exhibit fluctuations, leads to a complicated phenomenology [29,22]. Things are considerably simpler here, where the $\sigma$ field is trapped, and the situation for Jordan-Brans-Dicke theory was established in Ref. [10]. For the general scalar-tensor scenario we discuss, the relevant results can be found as a limiting case of the general analysis of Ref. 22].

The crucial parameters are the amplitude of the density perturbations, which, following 14.6 , we shall denote $\delta_{\mathrm{H}}$, the spectral index $n$ of the density perturbations and the contribution $R$ of gravitational waves to large angle microwave background anisotropies. In the Einstein frame, these are given by the standard formulae 14, 6

$$
\begin{aligned}
\delta_{\mathrm{H}} & =\frac{32}{75} \frac{V_{*}}{m_{\mathrm{Pl}}^{4}} \frac{1}{\epsilon_{*}} ; \\
n & =1-6 \epsilon_{*}+2 \eta_{*} ; \\
R & \simeq 12.4 \epsilon_{*},
\end{aligned}
$$

where subscript ' $*$ ' indicates that the quantities are to be evaluated when the relevant scales crossed outside the Hubble radius during inflation. The largest scales (such as the microwave background quadrupole) typically correspond to about $60 e$-foldings from the end of inflation, while the shortest scales (corresponding to galaxy formation) are at about 50 -foldings. So we can take ' $*$ ' to indicate $55 e$-foldings, the same as the bubbles, since the interesting $20 h^{-1} \mathrm{Mpc}$ scale for the bubbles is roughly in the middle of the large scale structure range.

The overall amplitude of perturbations can be fixed to match observations through scaling the potential by a suitable factor, thus determining $V_{0}$ (or $V_{0} / \Phi_{\text {end }}^{2}$ if there is a potential for $\Phi)$. The other two parameters can be constrained through compilation of large scale structure observations spanning as wide a range of scales as possible. This has been recently done for inflationary spectra by Liddle et al. [30], who considered the case of critical density, allowing arbitrary mixtures of cold dark matter and hot dark matter. The constraints can be summarized in terms of $\epsilon_{55}$ and $\eta_{55}$, and can be taken to be

$$
4 \epsilon_{55}-\eta_{55}<0.20 ; \quad \eta_{55}-\epsilon_{55}<0.10 \text {. }
$$

These are quite conservative, allowing the Hubble constant and the amount of hot dark matter to be freely chosen to allow the best possible fit. Specific choices for them would strengthen the constraints. The constraints map out a small area in the $\left(\epsilon_{55}, \eta_{55}\right)$ plane; in particular the maximum value of $\epsilon_{55}$ permitted by them is 0.10 (at $\left.\eta_{55}=0.20\right)$; for any higher value the amount of gravitational waves in the COBE signal is so high as to render the density perturbations too weak to give the observed structures.

\section{GRAPHICAL INTERPRETATION OF THE CONSTRAINTS}

We have found that the best way to illustrate the competing nature of these constraints is graphically, by plotting $\ln V$ against $N$, where $N$ is the number of $e$-foldings before the end of inflation. The reason is that one can rewrite $\epsilon$, using Eq. (8), as

$$
\epsilon=\frac{1}{2} \frac{\mathrm{d} \ln V}{\mathrm{~d} N}
$$

so $\epsilon$ simply gives the gradient of $\ln V(N)$. Furthermore, the curves corresponding to the original extended inflation model are straight lines in such a graph.

The bubble constraint immediately lets us constrain the mean $\epsilon$ over the last $55 e$-foldings, since

$$
\bar{\epsilon} \equiv \frac{1}{2} \frac{\Delta \ln V}{\Delta N}>0.10
$$

according to Eq. (17). This immediately shows the problem with the original extended inflation model; since $\epsilon$ 
is constant 0.10 is its minimum value, in conflict with the large scale structure requirement. Although as we have quoted it this seems very marginal, one should bear in mind that both sets of constraints are extremely conservative, especially the bubble one. A more accurate computation would create a significant gap between the two requirements.

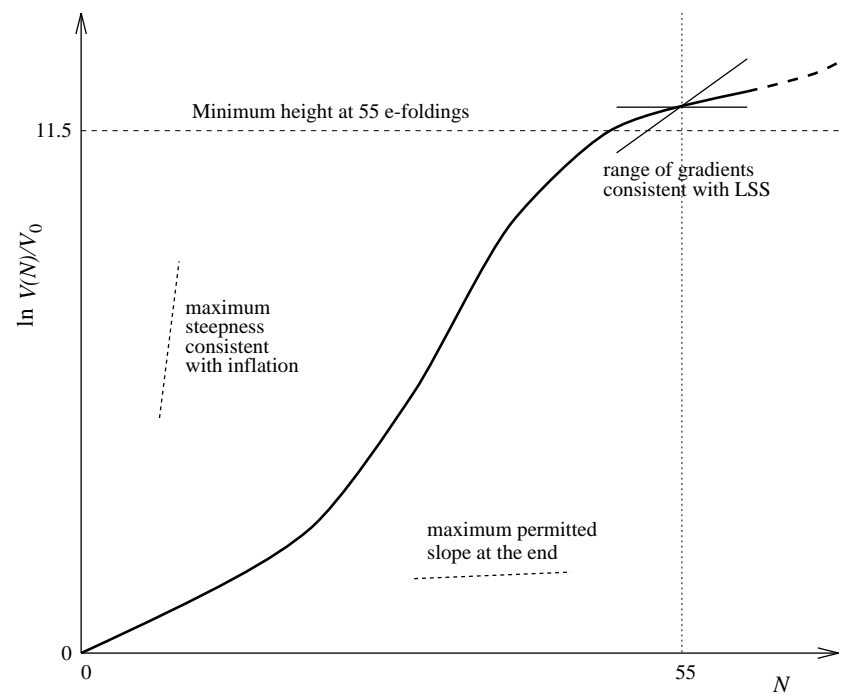

FIG. 1. A schematic diagram of the constraints, with gradients plotted roughly to scale. The short solid lines indicate the allowed gradient of the $\ln V(N)$ curve on the scales appropriate to large scale structure (LSS), assuming $\ln V(N)$ is linear. If its second derivative is significant, then the maximum allowed gradient becomes less, vanishing completely if $\ln V(N)$ becomes too curved. The short dotted lines schematically illustrate the slopes consistent with inflation and the maximum slope permitted at the end of inflation if the general relativity limit is to be obtained. The illustrated $\ln V(N)$ curve satisfies all constraints except the general relativity limit at the end of inflation.

Fig. 1 schematically illustrates all the constraints. At $55 e$-foldings from the end of inflation, the gradient of $\ln V(N) / V_{0}$ must be small enough to produce adequately scale-invariant density perturbations. If $\ln V(N)$ is linear, the gradient must be less than 0.2 . If $\ln V(N)$ has significant curvature, this too contributes to the breaking of scale-invariance and the maximum gradient is reduced, vanishing entirely if $\mathrm{d}^{2} \ln V / \mathrm{d} N^{2}$ is outside the range $(-0.02,0.01)$. Expressing $\mathrm{d}^{2} \ln V / \mathrm{d} N^{2}$ in terms of the slow-roll parameters

$$
\frac{\mathrm{d}^{2} \ln V}{\mathrm{~d} N^{2}}=4 \epsilon(\eta-2 \epsilon)
$$

allows us to restrict the values which $\mathrm{d} \ln V / \mathrm{d} N$ and $\mathrm{d}^{2} \ln V / \mathrm{d} N^{2}$ can simultaneously take, using the constraints on $\epsilon_{55}$ and $\eta_{55}$.

The bubble constraint dictates that at $55 e$-foldings, $\ln V(N) / V_{0}$ must be at least 11.5 , in order that the bubble constraint is satisfied; this requires a rapid decrease of $V$ as inflation proceeds. A linear extrapolation from the maximum allowed gradient, corresponding to an exponential potential, is insufficient to do the job, so the original extended inflation model (and any variant based on a constant $\omega$ ) is excluded. The $\ln V(N)$ curve must also descend respecting the condition for inflation, $\epsilon \lesssim 1$, which imposes a maximum gradient as illustrated. Finally, if general relativity is to be successfully attained after inflation without use of a potential for $\Phi$, then the gradient must be extremely shallow, as shown, at the end of inflation. A curve satisfying all these constraints yields a viable extended inflation model.

\section{SPECIFIC MODELS}

\section{A. Extended intermediate inflation}

Intermediate inflation [31] is an interesting model, because it is one of the rare inflation models which can give a 'blue' $(n>1)$ spectrum of density perturbations 32,33. Such spectra remain compatible with observational data [33, 30], provided $n$ is not too large. It can also give a scale-invariant spectrum [32]. Original versions of this model are not very satisfactory however, because there is no natural end to inflation. This can be rectified by implementing the model in our present framework; we can choose $\omega(\Phi)$ so as to give intermediate inflation in the Einstein frame, and have tunnelling to end inflation. Barrow and Maeda [18] found a class of such solutions, but at that time all the constraints on the scenario had not been discovered. García-Bellido and Wands 22 briefly indicated how to obtain the scaleinvariant $(n=1)$ intermediate inflation model in this way. The construction of viable models of this type was the original aim of our work; we now show that it cannot be realized.

The required coupling function $\omega(\Phi)$ is

$$
\omega(\Phi)+\frac{3}{2}=\frac{8 \pi}{m_{\mathrm{Pl}}^{2}} \frac{4}{\alpha^{2}} \beta^{2 / \alpha} \Phi^{4 / \alpha},
$$

where $\beta$ and $\alpha$ are dimensionless constants. From Eqs. (2) and (5), the Einstein frame potential has form $V(\phi)=\beta V_{0} \phi^{-\alpha}$, which is the potential whose slowroll solutions give intermediate inflation [31.32]. For $0<\alpha<2$ it produces a blue spectrum [32].

At large $V(\phi)$, the intermediate inflation potential is too steep to support inflation. Assuming that inflation commences when the potential becomes flat enough that $\epsilon=1$, Eq. (8) leads to an expression for $\phi$ at the end of inflation in terms of $\alpha$ and the total number of $e$-foldings $N_{\text {tot }}$, which is

$$
\phi_{\mathrm{end}}^{2}=\frac{\alpha m_{\mathrm{Pl}}^{2}}{16 \pi}\left(4 N_{\mathrm{tot}}+\alpha\right) .
$$

Using this to evaluate Eq. (14) at the end of inflation produces, in the approximation that $N_{\text {tot }} \gg \alpha$ and $\omega \gg$ 
1 , a relationship between the final value of $\omega, N_{\text {tot }}$ and $\alpha$, which is

$$
\omega(\Phi=1)=\frac{8 N_{\mathrm{tot}}}{\alpha} .
$$

Note that there are parameter values for which the solar system constraint on $\omega$ can be satisfied.

These expressions allow $V(\phi)$ to be written in a particularly simple form

$$
V(N)=V_{0}\left(1-\frac{N}{N_{\text {tot }}}\right)^{-\alpha / 2},
$$

so that

$$
\ln \frac{V(N)}{V_{0}}=-\frac{\alpha}{2} \ln \left(1-\frac{N}{N_{\text {tot }}}\right) .
$$

The gradient of $\ln V(N)$ increases with $N$ (that is, the curve as plotted in Fig. 1 curves upwards); this is a generic property of models in which $\omega$ is an increasing function of time. Since we have already seen that even a straight line (corresponding to power-law inflation) is excluded, this immediately means that if the amplitude of the potential is great enough to satisfy the bubble constraint, then the gradient evaluated over the range $50<N<60$ violates the constraints imposed by large scale structure. Indeed, the bubble constraint alone is sufficient to rule out intermediate inflation for the range of $\alpha$ values giving $n>1$.

The failure of this model immediately indicates how hard it will be to implement extended inflation models giving blue spectra, because from Eqs. (19), (22) and (24) the condition for $n>1$ is simply

$$
\frac{\mathrm{d}^{2} \ln V}{\mathrm{~d} N^{2}}>\left(\frac{\mathrm{d} \ln V}{\mathrm{~d} N}\right)^{2}
$$

implying that $\ln V(N)$ must be curving up at $55 e$ foldings. This could only give a model satisfying all the constraints if at lower values of $N$ it is curving down. This can only be achieved by using a potential (or equivalently $\omega(\Phi)$ ) which has many features during the late stages of inflation.

\section{B. General forms for $\ln V(N)$}

To end our discussion, we show that it is possible to construct models satisfying all the constraints, with the exception of the present value of $\omega$ (which could also be achieved by a suitably modified $\ln V(N)$ curve) which we shall assume is salvaged by a potential for $\Phi$. We investigate two general forms for $\ln V(N)$, identifying the range of parameter values for which the bubble constraint and the large scale structure constraints on the curvature of $\ln V(N)$ are simultaneously satisfied. Both these models are quite contrived, illustrating how difficult it is to remain within the presently existing constraints.
Given a particular form for $\ln V(N)$, it is possible to find $V(\phi)$ using the following relation, derived from Eq. (8), to obtain $\phi(N)$

$$
\phi-\phi_{\mathrm{end}}=\frac{m_{\mathrm{Pl}}}{\sqrt{8 \pi}} \int_{0}^{N}\left(\frac{\mathrm{d} \ln V}{\mathrm{~d} N}\right)^{1 / 2} \mathrm{~d} N,
$$

where, without loss of generality, we have assumed $\dot{\phi}$ positive.

Model 1: $\ln V(N) / V_{0}=a N-b N^{2}$

This model satisfies all the constraints for $0.23<$ $a<0.77$, with $b$ allowed to take a narrow range of small positive values, typically around $10^{-3}$, for a given $a$. The small negative quadratic term reduces the curvature of $\ln V(N) / V_{0}$ for large $N$ without significantly reducing its amplitude.

In terms of $\phi$, this potential has form

$$
\ln \frac{V(\phi)}{V_{0}}=\frac{a^{2}}{4 b}\left[1-\left(1-\frac{\sqrt{72 \pi} b}{a^{3 / 2}} \frac{\phi_{\mathrm{end}}-\phi}{m_{\mathrm{Pl}}}\right)^{4 / 3}\right] .
$$

Model 2: $\ln V(N) / V_{0}=A \sin \left(\pi N / 2 N_{\max }\right)$

For this rather unnatural model, values of $A$ between 11.8 and 22.5 are allowed; however the values of $N_{\text {max }}$ permitted for any given $A$ are strongly limited by the large scale structure constraints on the gradient and curvature of $\ln V(N) / V_{0}$. For $11.8<A<16.5$ the region of allowed values of $N_{\text {max }}$ forms a single band, with a lower limit of 63. However for $16.5<A<20$ this splits into two narrow bands and finally for $20<A<22.5$ only a small range of values around $N_{\max } \approx 145$ are allowed.

\section{CONCLUSIONS}

We have carried out a model-independent analysis of the constraints on extended inflation scenarios containing a trapped scalar field within an arbitrary scalar-tensor theory. We have shown that all of the constraints can be interpreted graphically, by considering the behaviour of the (logarithm of the) Einstein frame potential as a function of the number of $e$-foldings from the end of inflation. The principal competition arises from the need to keep the density perturbation spectrum adequately scale-invariant while suppressing the production of bubbles which finish with astrophysically large sizes.

Especially bearing in mind that our implementation of the constraints is quite conservative, we have been able to show how difficult it is to obtain a successful extended inflation scenario. Indeed, all models of this type which presently exist in the literature, in which bubble 
nucleation ends inflation, cannot evade the combination of constraints. Things seem particularly tough if one desires a 'blue' spectrum of perturbations, which is a situation in which one would have hoped extended inflation might have fared well since such models are hard to implement in the chaotic inflation framework. Our graphical approach allows one to see exactly what is needed to obtain working models; for example, any model in which $\omega$ monotonically increases with time will not work. We have devised models which are allowed, though they seem rather contrived.

To conclude, the extended inflation paradigm is an attractive one, because the new physics, that of extended gravitational theories, can be tested in a number of ways. Unfortunately, when one also adds the extra constraints brought on by demanding that inflation ends by a firstorder transition, the scenario becomes so highly constrained that it is extremely hard to find any working models. Still, the fact that one can exclude inflationary models on the basis of observational data should be viewed as an encouraging situation, and one we shall hear much of in future years.

\section{ACKNOWLEDGEMENTS}

AMG is supported by PPARC and ARL by the Royal Society. We thank Juan García-Bellido and David Wands for their comments, and acknowledge use of the Starlink computer system at the University of Sussex.

\section{APPENDIX: RELATIONS BETWEEN THE JORDAN AND EINSTEIN FRAMES}

Some aspects of the relation between the frames have been considered by Lidsey 34. We shall use the slowroll parameters $\epsilon$ and $\eta$ to generalize those arguments, producing exact relationships between Einstein and Jordan frame quantities as well as investigating the slow-roll limit.

\section{Condition for Inflation}

Inflation corresponds to an accelerating scale factor. However, it is possible for the scale factor to be accelerating in one frame and not the other. The inflationary condition can be rewritten as

$$
\frac{\mathrm{d} H}{\mathrm{~d} t}+H^{2}>0
$$

(and similarly in the Einstein frame) which is more convenient for comparison between the Einstein and Jordan frames.

There is an exact relation given by

$$
\frac{1}{a} \frac{\mathrm{d}^{2} a}{\mathrm{~d} t^{2}}=\Omega^{2}\left(\frac{\mathrm{d} \tilde{H}}{\mathrm{~d} \tilde{t}}+\tilde{H}^{2}\right)-\Omega \frac{\mathrm{d} \Omega}{\mathrm{d} \tilde{t}} \tilde{H}+\left(\frac{\mathrm{d} \Omega}{\mathrm{d} \tilde{t}}\right)^{2}-\Omega \frac{\mathrm{d}^{2} \Omega}{\mathrm{d} \tilde{t}^{2}}
$$

and using Eq. (9) we obtain

$$
\begin{aligned}
\frac{1}{a} \frac{\mathrm{d}^{2} a}{\mathrm{~d} t^{2}}= & \Phi\left[\left(\frac{\mathrm{d} \tilde{H}}{\mathrm{~d} \tilde{t}}+\tilde{H}^{2}\right)-\frac{\sqrt{\pi \epsilon}}{m_{\mathrm{Pl}}}\left(\frac{\mathrm{d} \phi}{\mathrm{d} \tilde{t}} \tilde{H}-\frac{\mathrm{d}^{2} \phi}{\mathrm{d} \tilde{t}^{2}}\right)\right] \\
& -\Phi\left[\frac{8 \pi}{m_{\mathrm{Pl}}^{2}} \frac{\eta-2 \epsilon}{8}\left(\frac{\mathrm{d} \phi}{\mathrm{d} \tilde{t}}\right)^{2}\right]
\end{aligned}
$$

The slow-roll approximation allows us to systematically discard terms, and leads, to first order in the slow-roll parameters, to the relation

$$
\frac{1}{a} \frac{\mathrm{d}^{2} a}{\mathrm{~d} t^{2}}=\Phi\left[\left(\frac{\mathrm{d} \tilde{H}}{\mathrm{~d} \tilde{t}}+\tilde{H}^{2}\right)+\frac{2 \pi}{3 m_{\mathrm{Pl}}^{2}} \epsilon V\right] .
$$

The right hand side of this equation is always positive, so inflation in the Einstein frame guarantees inflation in the Jordan frame, as found by Lidsey [34.

\section{Number of $e$-foldings}

The number of $e$-foldings of inflation in the two frames, given by

$$
N=\int H \mathrm{~d} t \quad ; \quad \tilde{N}=\int \tilde{H} \mathrm{~d} \tilde{t},
$$

are in general different. However, from Eqs. (13) and (14) we can see that they are approximately equal provided

$$
\left|\frac{\mathrm{d} \Omega / \mathrm{d} \tilde{t}}{\Omega \tilde{H}}\right| \ll 1
$$

With the use of Eqs. (2), (6) and (9), this becomes simply

$$
\epsilon \ll 2 \text {. }
$$

Hence in the slow-roll limit the amount of inflation as measured in the different frames coincides.

[1] D. La and P. J. Steinhardt, Phys. Rev. Lett. 62, 376 (1989).

[2] E. W. Kolb, Physica Scripta T36, 199 (1991).

[3] A. H. Guth, Phys. Rev. D23, 347 (1981).

[4] A. D. Linde, Phys. Lett. B129, 177 (1983); A. D. Linde, Particle Physics and Inflationary Cosmology, Harwood Academic (Chur, Switzerland, 1990). 
[5] E. W. Kolb and M. S. Turner, The Early Universe, (Addison-Wesley, Redwood City, California, 1990).

[6] A. R. Liddle and D. H. Lyth, Phys. Rep. 231, 1 (1993).

[7] C. M. Will, Theory and Experiment in Gravitational Physics, Cambridge University Press (Cambridge, 1993).

[8] E. J. Weinberg, Phys. Rev. D40, 3950 (1989).

[9] D. La, P. J. Steinhardt and E. Bertschinger, Phys. Lett. B231, 231 (1989).

[10] E. W. Kolb, D. S. Salopek and M. S. Turner, Phys. Rev. D42, 3925 (1990); A. H. Guth and B. Jain, Phys. Rev. D45, 426 (1992); D. H. Lyth and E. D. Stewart, Phys. Lett. B274, 168.

[11] A. H. Guth and S.-Y. Pi, Phys. Rev. Lett. 49, 1110 (1982); A. A. Starobinsky, Phys. Lett. B117, 175 (1982); S. W. Hawking, Phys. Lett. B115, 295 (1982); J. M. Bardeen, P. J. Steinhardt and M. S. Turner, Phys. Rev. D28, 679 (1983).

[12] C. Brans and R. H. Dicke, Phys. Rev. 24, 925 (1961).

[13] R. D. Reasenberg et.al., Astrophys.J. 234, L219 (1979).

[14] A. R. Liddle and D. H. Lyth, Phys. Lett. B291, 391 (1992).

[15] A. R. Liddle and D. H. Lyth, Ann. N. Y. Acad. Sci 688, 653 (1993).

[16] P. J. Steinhardt and F. Accetta, Phys. Rev. Lett. 64, 2740 (1990); R. Crittenden and P. J. Steinhardt, Phys. Lett. B293, 32 (1992).

[17] F. S. Accetta and J. J. Trester, Phys. Rev. D39, 2854 (1989); R. Holman, E. W. Kolb and Y. Wang, Phys. Rev. Lett. 65, 17 (1990); J. García-Bellido and M. Quirós, Phys. Lett. B243, 45 (1990); R. Holman, E. W. Kolb, S. Vadas and Y. Wang, Phys. Rev. D43, 3833 (1991); R. Holman, E. W. Kolb, S. Vadas and Y. Wang, Phys. Lett. B269, 252 (1991); R. Crittenden and P. J. Steinhardt, Phys. Lett. B293, 32 (1992); A. Laycock and A. R. Liddle, Phys. Rev. D49, 1827 (1994); F. Occhionero and L. Amendola, Phys. Rev. D50, 4846 (1994).

[18] J. D. Barrow and K. Maeda, Nucl. Phys. B341, 294 (1991).

[19] Y. Wang, Phys. Rev. D44, 991 (1991).

[20] A. R. Liddle and D. Wands., Phys. Rev. D45, 2665 (1992).

[21] K. Maeda, Phys. Rev. D39, 3159 (1989).

[22] J. García-Bellido and D. Wands, Phys. Rev. D52, 6739 (1995).

[23] H. Nariai, Prog. Theor. Phys. 42, 544 (1969).

[24] F. S. Accetta, L. M. Krauss and P. Romanelli, Phys. Lett. B248, 146 (1990); J. A. Casas, J. García-Bellido and M. Quirós, Phys. Lett. B278, 94 (1992).

[25] A. H. Guth and E. J. Weinberg, Nucl. Phys B212, 321 (1983).

[26] A. Wu, Phys. Rev. D45, 2653 (1992).

[27] A. R. Liddle and D. Wands, Mon. Not. Roy. Astron. Soc. 253, 637 (1991); A. R. Liddle and D. Wands, Phys. Lett. B276, 18 (1992).

[28] E. J. Copeland, A. R. Liddle, D. H. Lyth, E. D. Stewart and D. Wands, Phys. Rev D49, 6410 (1994).

[29] A. A. Starobinsky and J. Yokoyama, Kyoto preprint (1995), gr-qc/9502002.

[30] A. R. Liddle, D. H. Lyth, R. K. Schaefer, Q. Shafi and P. T. P. Viana, to appear, Mon. Not. Roy. Astron. Soc.
(1996), astro-ph/9511057.

[31] J. D. Barrow, Phys. Lett. B249, 406 (1990); A. G. Muslimov, Class. Quant. Grav 7, 231 (1990); J. D. Barrow and P. Saich, Phys. Lett. B249, 406 (1990).

[32] J. D. Barrow and A. R. Liddle, Phys. Rev. D47, R5219 (1993).

[33] S. Mollerach, S. Matarrese and F. Lucchin, Phys. Rev. D50, 4835 (1994).

[34] J. E. Lidsey, Class. Quant. Grav. 9, 149 (1992). 